\title{
APPROXIMATE UNITS IN IDEALS OF GROUP ALGEBRAS
}

\author{
HARALD RINDLER
}

\begin{abstract}
Several properties of locally compact groups can be characterized by the existence of certain approximate units in 3 ideals of the group algebra. We combine methods and results from [8], [7], [5].
\end{abstract}

Let $G$ be a locally compact group, $M(G)$ the Banach algebra of all bounded complex measures, $L^{1}(G)$ the ideal consisting of all absolutecontinuous measures with respect to left Haar-measure,

$$
M^{0}(G)=\left\{m \in M(G), \int 1 d m=0\right\}, \quad L^{0}(G)=M^{0}(G) \cap L^{1}(G) .
$$

Let $B$ be a Banach algebra. We consider the following properties: (U) $B$ has a unit, (A) there exists a bounded net $u_{\alpha}$ in $B$ such that $\lim _{\alpha} u_{\alpha} * f=f$ for every $f \in B,\left(\mathrm{~A}_{c}\right)$ in addition $u_{\alpha} \in Z(B)$ the center of $B,\left(\mathrm{~A}_{d}\right)\left(u_{\alpha} * f-f\right) \in$ $Z(B)$ for every $f \in B$.

We consider the following classes of locally compact groups: [SIN]: $G$ has a basis of neighbourhoods of 1 invariant under all inner automorphisms, $[\mathrm{FC}]^{-}$: every conjugacy class in $G$ is precompact, $[\mathrm{FIA}]^{-}$: the group of inner automorphisms is precompact in the group of all continuous automorphisms, $[\mathrm{FD}]^{-}$, resp. FD: the group generated by all commutators $\left(x y x^{-1} y^{-1}\right)$ is precompact, resp. finite (see [3]). $G$ is amenable if there exists a left invariant mean on $L^{\infty}(G)$, the space of all measurable essentially bounded functions (see [2] or [8]).

It is well known that $L^{1}(G)$ always satisfies (A), and (U) iff $G$ is discrete. Mosak, [7], proved that $L^{1}(G)$ satisfies $\left(\mathrm{A}_{c}\right)$ iff $G \in$ [SIN]. Reiter, [9], proved that $L^{0}(G)$ satisfies (A) iff $G$ is amenable and Kotzmann, Rindler, [5], that $L^{0}(G)$ satisfies $\left(\mathrm{A}_{c}\right)$ iff $G \in[\mathrm{FIA}]^{-}$.

Using their methods and results and well-known structure theorems we easily can complete the results above to the following list of characterizations:

Let $G$ be a locally compact group then
1. $G$ is discrete
iff $\quad L^{1}(G)$ satisfies $U$
2. $G$ is compact
iff $\quad M^{0}(G)$ satisfies $\mathrm{U}$
3. $G$ is finite
iff
$L^{0}(G)$ satisfies $\mathrm{U}$

Received by the editors September 1, 1977.

AMS (MOS) subject classifications (1970). Primary 43A20, 22D05, $22 \mathrm{D15}$.

Key words and phrases. Locally compact group, group algebra, approximate unit, amenable group, compactness conditions. 

4. $\quad G$ is arbitrary l.c.
then $L^{1}(G)$ satisfies A
5. $G$ is amenable
iff $\quad M^{0}(G)$ satisfies A
6. $G$ is amenable
iff
$L^{0}(G)$ satisfies A
7. $G \in[\mathrm{SIN}]$
iff
$L^{1}(G)$ satisfies $\mathrm{A}_{c}$
8. $G \in[\mathrm{FC}]^{-}$
iff
$M^{0}(G)$ satisfies $\mathrm{A}_{c}$
9. $G \in[\mathrm{FIA}]^{-}$
iff $\quad L^{0}(G)$ satisfies $\mathrm{A}_{c}$
10. $G$ is Abelian or discrete iff $L^{1}(G)$ satisfies $\mathrm{A}_{d}$
11. $G \in[\mathrm{FD}]^{-}$
iff $M^{0}(G)$ satisfies $\mathrm{A}_{d}$
12. $G$ is Abelian or discrete iff $L^{0}(G)$ satisfies $\mathrm{A}_{d}$. and $\in[\mathrm{FD}]$

RemarKs. If the boundedness assumption of the net $u_{\alpha}$ is dropped then the following remarks apply: If we only assume that for every $f \in B$ and $\varepsilon^{*}>0$ there exists $u=u(f, \varepsilon) \in B\|u * b-u\|<\varepsilon$, then parts 5 and 6 remain open. The case of compact extensions of connected groups was settled in [10, Theorem 1 and Proposition 1]. A counterexample for discrete groups would lead to a nonamenable group not containing the free group of 2 generators, $B$. E. Johnson, [4]. If $u=u(f, \varepsilon)$ can be taken bounded independent of $f$ and $\varepsilon$ then (A) holds (see Altman [1] or Wichmann [12] who also proved that the same bound can be chosen for the net $u_{\alpha}$ ). For $M^{0}(G)$ and $L^{0}(G)$ the best possible bound is 2 .

Proofs. If $\delta$ is the unit of $M(G), \lambda$ the normalized Haar measure if $G$ is compact (resp. finite) then $\delta-\lambda$ is a unit of $M^{0}(G)$ (resp. $L^{0}(G)$ ). The proof of the converse in 3 contains proofs of 1 and 2: If $u \in L^{0}(G)$ satisfies $f * u=f$ for every $f \in L^{0}(G)$ we obtain for $m=\delta-u, \int 1 d m=1$ and $f * m=0, f \in L^{0}(G)$. Choosing $f(z)=g(x z)-g(z), g$ continuous with compact support, $x \in G$ arbitrary, it follows from $0=f * m(e)=$ $\int f(y) d m\left(y^{-1}\right)$ that $m$ is a right Haar measure. $\int 1 d m=1$ implies $G$ is compact, $m$ is absolutely continuous and so is $\delta=u+m$ and $G$ is also discrete. If $u$ is a unit for $M^{0}(G)$ then in the same way we obtain that $G$ is compact. If $u$ is a unit for $L^{1}(G)$ then $0=f *(\delta-u)(e)$, for every $f$ continuous with compact support implies that $u=\delta \in L^{1}(G)$ and we obtain that $G$ is discrete. The converse is clear.

The proof of 6 was given in [9], 5 follows analogously. The proof of 7 is contained in [7], that of 9 in [5].

Proof of 8. If $\lim \left\|u_{\alpha} * m-m\right\|=0$ for every $m \in M^{0}(G), u_{\alpha}$ central, then $v_{\alpha}=\delta-u_{\alpha}$ is central and $v_{\alpha} * m \rightarrow 0$ for every $m \in M^{0}(G)$. If $w_{\alpha}$ denotes the absolutely continuous part of $v_{\alpha}$, then $w_{\alpha}$ also belongs to the center of $M(G)$ and putting $m=g-\delta, g \in L^{1}(G), \int g=1$, we obtain $\left\|w_{\alpha}-v_{\alpha}\right\| \leqslant\left\|v_{\alpha} *(g-\delta)\right\| \rightarrow 0 \quad\left(v_{\alpha} * g \in L^{1}(G)\right)$. This implies $\lim _{\alpha}\left\|w_{\alpha} * m\right\|=0$ for every $m \in M^{0}(G)$, therefore $G \in[\mathrm{FC}]^{-}$by [5, Proposition, (c)]. 
If conversely $G \in[\mathrm{FC}]^{-}$then by Robertson's structure theorem ([11], for a proof see [6]) there exists a compact normal subgroup $K$ such that $G / K=V$ $\times D, V$ a vector group, $D$ a discrete [FC]-group. This reduces the problem to discrete groups, but then $M^{0}(G)=L^{0}(G)$ and the result follows from 9 .

To prove the nontrivial part of 10 we assume that $\left(\delta-u_{\alpha}\right) * f$ is central for every $f \in L^{1}(G)$. Choosing $f_{\beta}$ the normalized characteristic functions of $U_{\beta}$, a basis of neighbourhoods of $x \in G$, we obtain that $\left(\delta-u_{\alpha}\right) * \delta_{x}$ is central for every $x \in G$ (the center being weakly closed). The discrete part of this measure is also central and equal to $\delta_{x}$ if $G$ is not discrete and $G$ is necessarily Abelian.

If $G \in$ [FD $^{-}$then there exists a compact normal subgroup $K$ such that $G / K$ is Abelian. We obtain a suitable approximate unit for $M^{0}(G)$ by lifting from $M^{0}(G / K)$. Conversely we have $\left(\delta-u_{\alpha}\right) * m$ is central for every $m \in$ $M^{0}(G)$. For a fixed $\alpha_{0}$ we put $\mu=\delta-u_{\alpha_{0}}$. We want to show that $\mu * m$ is central for every $m \in M(G)$. We may assume that $\int 1 d m=1$, then $(m-$ $\left.u_{\alpha}\right) \in M^{0}(G)$ and $\mu *\left(m-u_{\alpha}\right)$ is central for every $\alpha$. Furthermore $\lim _{\alpha} \mu *$ $u_{\alpha}=\lim _{\alpha} u_{\alpha} * \mu=0$. The center being closed this implies that $\mu * m$ and in particular $\mu * \delta_{x}$ is central for every $x \in G$. This implies that $\mu=\mu * \delta_{x y x^{-1} y-1}$ and $\mu=\mu * \delta_{z}$ for every $z \in K$ the closure of the commutator subgroup. $\mu$ being a bounded measure, this implies that $G$ is compact.

ProOF OF 12. As before, we obtain that $\left(\delta-u_{\alpha}\right) * \delta_{x}$ is central for all $x \in G$. By 10 we may assume that $G$ is discrete and by $11 G$ is a [FC]-group. The converse is clear.

\section{REFERENCES}

1. M. Altman, Contracteurs dans les algèbres de Banach, C. R. Acad. Sci. Paris Sér. A-B 274 (1971), A399-A400. MR 45 \#2437.

2. P. F. Greenleaf, Invariant means of topological groups, Van Nostrand, New York, 1969. MR 40 \# 4776.

3. S. Grosser and M. Moskowitz, Compactness conditions in topological groups, J. Reine Angew. Math. 246 (1971), 1-40. MR 44 \# 1766.

4. B. E. Johnson, Some examples in harmonic analysis, Studia Math. 48 (1973), 182-188. MR 49 \#3456.

5. E. Kotzmann and H. Rindler, Central approximate units in a certain ideal of $L^{1}(G)$, Proc. Amer. Math. Soc. 57 (1976), 155-158. MR 53 \#8784.

6. J. Liukkonen, Dual spaces of groups with precompact conjugacy classes, Trans. Amer. Math. Soc. 180 (1973), 85-108. MR 47 \#6937.

7. R. D. Mosak, Central functions in group algebras, Proc. Amer. Math. Soc. 29 (1971), 613-616. MR 43 \#5323.

8. H. Reiter, Classical harmonic analysis and locally compact groups, Clarendon Press, Oxford, 1968. MR 46 \#5933.

9. A882-A885. MR 39 \#6025.

10. H. Rindler, Zur Eigenschaft $P_{1}$ lokalkompakter Gruppen, Indag. Math. 35 (1973), 142-147. MR 48 \# 11921.

11. L. C. Robertson, A note on the structure of Moore groups, Bull. Amer. Math. Soc. 75 (1969), 594-599. MR 39 \# 7027.

12. J. Wichmann, Bounded approximate units and bounded approximate identities, Proc. Amer. Math. Soc. 41 (1973), 547-550. MR 48 \#2767.

Mathematics Institute, University of VienNa, A-1090 VienNa, AUstria 